\begin{tabular}{|c|c|c|c|c|c|c|}
\hline \multirow{4}{*}{ Impact Factor: } & ISRA (India) & $=3.117$ & SIS (USA) & $=0.912$ & ICV (Poland) & $=6.630$ \\
\hline & ISI (Dubai, UAE & $=0.829$ & РИНЦ (Russia) & $=0.156$ & PIF (India) & $=1.940$ \\
\hline & GIF (Australia) & $=0.564$ & ESJI $(\mathrm{KZ})$ & $=8.716$ & IBI (India) & $=4.260$ \\
\hline & JIF & $=1.500$ & SJIF (Morocco) & $=5.667$ & OAJI (USA) & $=0.350$ \\
\hline
\end{tabular}

\begin{tabular}{|c|c|}
\hline $\begin{array}{l}\text { SOI: } \frac{1.1 / \mathrm{TA}}{} \\
\text { International Sc } \\
\text { Theoretical } \mathbb{\&} \mathrm{A}\end{array}$ & $\begin{array}{l}\text { AS DOI: } 10.15863 / \mathrm{TAS} \\
\text { cientific Journal } \\
\text { Applied Science }\end{array}$ \\
\hline p-ISSN: 2308-4944 (print) & e-ISSN: 2409-0085 (online) \\
\hline Year: 2019 Issue: 06 & Volume: 74 \\
\hline Published: 15.06.2019 & http://T-Science.org \\
\hline
\end{tabular}

SECTION 3. Nanotechnology. Physics.
QR - Issue

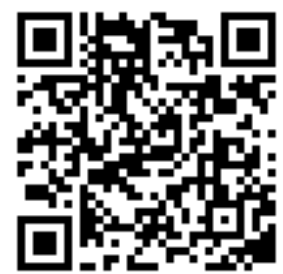

UDC 53.02

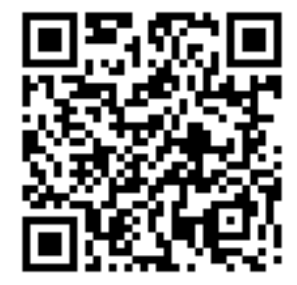

Doolotbai Babaev

International Kuwait University doctor of pedagogical sciences, professor Bishkek, Kyrgyz Republic

Minavar Matkarimova the Jalal-Abad State University Lecturer, Jalal-Abad, Kyrgyz Republic

Shavkat Kimsanbaevich Haitov Kyrgyz-Uzbek University Senior lecturer, Osh, Kyrgyz Republic

\title{
THE ROLE OF PHYSICS SUBJECT IN TEACHING COLLEGE STUDENTS
}

Abstract: The article discusses the role of physics and mathematics in colleges. And also some examples of solving physical problems of an applied nature are given. Disclosed interdisciplinary communication subject of mathematics and physics.

Key words: training, information technology, interactive learning, interdisciplinary communication.

Language: Russian

Citation: Babaev, D., Matkarimova, M., \& Haitov, S. K. (2019). The role of physics subject in teaching college students. ISJ Theoretical \& Applied Science, 06 (74), 221-226.

Soi: http://s-o-i.org/1.1/TAS-06-74-24 Doi: crostef https://dx.doi.org/10.15863/TAS.2019.06.74.24

\section{РОЛЬ ПРЕДМЕТА ФИЗИКИ ПРИ ОБУЧЕНИИ СТУДЕНТОВ КОЛЛЕДЖА}

Аннотация: В статье рассмотрена роль физики и математики в колледжах. А также приведены некоторые примеры решения физических задач прикладного характера. Раскрыты межпредметные связи предмета математики и физики.

Ключевые слова: профессиональная подготовка, информационные технологии, интерактивное обучение, межпредметный связь.

\section{Introduction}

Перед вузами и колледжами Кыргызстана в данное время ставятся новые задачи, направленные на формирование у студентов системного мышления, творческой активности и самостоятельности. В колледжах по подготовке будущих специалистов основными функциями являются не усвоение готовых знаний, которые получены во время лекционных и практических занятиях по физике, а развитие у студентов способностей к овладению методами научного познания, дающими возможность самостоятельно получить знания и творческое использование на основе знакомых методов профессиональной деятельности. Изучение предмета физики в колледжах способствует не только повышению естественно-математического уровня подготовки студентов, но также формирует у них творческое мышление. В своей будущей профессиональной деятельности студенты столкнуться с необходимостью решить проблемные ситуации, которые требуют своеобразный подход. "Проблемная ситуация - это особый вид мыслительного воздействия объекта и субъекта, который характеризуется таким психологическим состоянием, возникающим у субъекта (учащегося) при выполнении задания, которое требует найти (освоить) новые, ранее неизвестные субъекту знания и способы действия". Проблемная ситуация заранее конструируется преподавателем, в основе ее лежит учебная проблема. При ее реализации обучаемый вынужден самостоятельно 


\begin{tabular}{llllll} 
& ISRA (India) $=\mathbf{3 . 1 1 7}$ & SIS (USA) & $=\mathbf{0 . 9 1 2}$ & ICV (Poland) & $=\mathbf{6 . 6 3 0}$ \\
Impact Factor: & ISI (Dubai, UAE) $=\mathbf{0 . 8 2 9}$ & PUHL (Russia) $=\mathbf{0 . 1 5 6}$ & PIF (India) & $=\mathbf{1 . 9 4 0}$ \\
& GIF (Australia) $=\mathbf{0 . 5 6 4}$ & ESJI (KZ) & $=\mathbf{8 . 7 1 6}$ & IBI (India) & $=\mathbf{4 . 2 6 0}$ \\
& JIF & $\mathbf{1 . 5 0 0}$ & SJIF (Morocco) $=\mathbf{5 . 6 6 7}$ & OAJI (USA) & $\mathbf{0 . 3 5 0}$ \\
\hline
\end{tabular}

добывать новые знания [1]. Продуктивное решение таких проблем будет недопустимо без конкретного опыта творческого труда.

\section{Materials and Methods}

В настоящее время совершенствование преподавания фундаментальных дисциплин является одним из важнейших направлений развития Кыргызской Республики [4]. Инновационное производство является актуальной для одной из важнейших отраслей экономики Кыргызстана - отрасли выработки электрической энергии. В «Стратегия развития образования в Кыргызской Республике на 20122020 годы» определены актуальные проблемы среднего профессионального образования и приоритетные направления политики. Среди актуальных проблем среднего профессионального образования необходимо выделить следующие [6]:

1) Несоответствие результатов среднего профессионального образования (СПО) требованиям, предъявляемым рынком труда. Существующие квалификационные требования в СПО устарели и поэтому выпускники не обладают навыками и знаниями, востребованными на рынке труда. Низкое качество программ СПО объясняется недостаточностью систематического взаимодействия между учреждениями СПО, частным сектором (бизнес) и рынком труда. Учебные программы СПО больше ориентированы на теорию, а не на практические компетенции.

2) Неконкурентоспособные выпускники системы СПО по ряду специальностей и профессий. Профессии и специальности в СПО не анализируются регулярно с точки зрения перспективных требований развивающегося рынка труда. Поэтому, в дополнение к слабой подготовке выпускников система, в силу негибкости, сталкивается с проблемой переизбытка или недостатка определенных профессий.

Исходя вышеперечисленных проблем нами поставлена цель - подготовка специалистов, способных реализовать профессиональную деятельность в сфере производства и в быту, учитывая процесс преподавания предмета физики. Изучение физики в колледжах дает возможность формированию у студентов фундаментальные физические знания, т.е. развитие технические способности и технические мышления, которые обеспечивает плодотворное решение технических задач.

После окончания школы ученики поступают в вузы и колледжи, имеющие свои требования и организации учебного процесса. В период первого семестра многие студенты проходит благополучно, привыкая к новой атмосфере общества. Для преподавателей фундаментальных дисциплин, т.е. физики и математики, важно развивать творческое мышление. Для осуществления данной деятельности в процессе обучения можно применять некоторые методы и технологии преподавания. Для повышения полученных знаний в колледжах, очень важно опираться на ранее полученные знания, т.е. должен реализоваться принцип преемственности. При этом сравнивается знании по физике общеобразовательной школы и колледжа, которое способствует формированию основных законов физики [2].

Основной задачей университетов и колледжей является профессиональная подготовка студентов, но творческая составляющая в этом процессе необходима. Аналогичное развитие с помощью предмета физики позволяет достигать следующие результаты:

развивается аналитические способности, совершенствуются логические процессы;

- для нахождения новых решений старых задач создаются основа;

- развивается критическое мышление;

- создается более комфортная обстановка в процессе обучения предмета физики.

Для этого необходимо совершенствовать преподавания физики в колледжах, т.е. надо использовать инновационные педагогические технологии в процессе преподавания физики.

Под инновациями в образовании понимается процесс совершенствования педагогических технологий, совокупности методов, приемов и средств обучения. Основную задачу педагогической деятельностью является создании на занятиях по физике такой образовательной среды, которое дает возможность самореализации студентов, повышению их уровня знаний, формированию коммуникативных навыков, творческого мышления, познавательной активности.

Применение активных и интерактивных методов обучения повышает интерес к знаниям. В практике можно использовать такие педагогические технологии обучения как [3]:

- технология проблемного обучения стимулировать студентов к самостоятельному получению знаний, необходимых для решения проблемы прикладного характера;

- информационно-коммуникационные технологии применяются на занятиях при объяснении нового материала, при закреплении, для контроля знаний, при выполнении домашнего задания, при подготовке к экзаменам; 


\begin{tabular}{llllll} 
& ISRA (India) $=\mathbf{3 . 1 1 7}$ & SIS (USA) & $=\mathbf{0 . 9 1 2}$ & ICV (Poland) & $=\mathbf{6 . 6 3 0}$ \\
Impact Factor: & ISI (Dubai, UAE) $=\mathbf{0 . 8 2 9}$ & PUHL (Russia) $=\mathbf{0 . 1 5 6}$ & PIF (India) & $=\mathbf{1 . 9 4 0}$ \\
& GIF (Australia) $=\mathbf{0 . 5 6 4}$ & ESJI (KZ) & $=\mathbf{8 . 7 1 6}$ & IBI (India) & $=\mathbf{4 . 2 6 0}$ \\
& JIF & $\mathbf{1 . 5 0 0}$ & SJIF (Morocco) $=\mathbf{5 . 6 6 7}$ & OAJI (USA) & $\mathbf{0 . 3 5 0}$ \\
\hline
\end{tabular}

- личностно-ориентированные технологии;

- технология проектного обучения индивидуальная или коллективная деятельность по отбору, распределению и классификация материала по конкретной теме, в результате которой разрабатывается научный проект.

Мотивирование студентов к активной мыслительной деятельности является мотивирование - совершенствование организации учебного процесса и предложить студентам нетрадиционные формы занятия[5]:

- урок-семинар - при изучении темы "Тепловые двигатели проводится урок-семинар, группа предварительно на подгруппы и объясняется общий план подготовки (определение, устройство, принцип действия, применение, влияние на окружающую среду) по разным типам двигателей. Заданий такого характера студенты выполняют с удовольствием, показывают свои активности, и урок - семинар проходит оживленно и более интересным;

- урок защиты проектных работ - для развития навыков проектной деятельности студентам предлагается мини-сочинения: «Для чего нужна изучать физику?», «Физика в быту», «Оптические явления», «Оптические приборы», «Использование электродвигателей постоянного тока»;

- урок-соревнование - проведение занятий по теме «Законы постоянного электрического тока», в котором студенты будут соревноваться по числу набранных балов по итогу занятия.

- урок-экскурсия - это важный прием не только повышающий эффективность усвоение материала в целом, но и проявляет интерес студентов к фундаментальным наукам;

- виртуальные интерактивные лабораторные работы предоставляет уникальную возможность визуализации моделей физических явлений и процессов. Интерактивность открывает перед студентами большие возможности, делая их не только наблюдателями, но и активными участниками проводимых экспериментов.

Важной формой развития интереса студентов к физике и их творческих способностей является решение физических задач прикладного характера. Для укрепления знаний необходимо не физические задачи, а наоборот, решить физические задачи прикладного характера нужны знании. Основными компонентами специалиста (электроснабжение, техническое обслуживание и ремонт автомобильных транспорта) является решение задач, т.е. принять оптимальных решений поставленной цели в заданных условиях. Поэтому в вузах и колледжах Кыргызстана процесс преподавания физики должен быть направлен на решение физических задач, используя математические аппараты. Реализация в учебный процесс задач прикладного характера способствует укреплению знаний по физике и математике.

В русле вышеизложенных, необходимо осуществлять межпредметные связи «математикафизика» необходимо соблюдать чувство меры: уроки математики не должны превращаться в уроки физики с применением математики, нельзя перейти черту границ этих предметов и загромождать текст прикладной задачи такими техническими и статистическими данными, которые порождают у учащихся дополнительные трудности.

На уровне знаний раскрываются межпредметные связи по направлению математика и физика» и в следующей задаче.

Задача 1: Элемент замыкается спиралью один раз сопротивлением $R_{1}$, другой раз сопротивлением $R_{2}$. В том и другом случае количество тепла, выделяющегося в спиралях за одно и то же время, оказывается одинаковым. Каково внутреннее сопротивление $r$ ?

Решение.

I - этап (формализация). Построим математическую модель явления. По условию задачи имеем: $Q_{1}=Q_{2}$, где $Q_{1}$ - количество тепла, выделяющегося в первой спирали, $Q_{2}$ количество тепла, выделяющегося во второй спирали. Поскольку $Q_{1}=I_{1}^{2} R_{1} t$, а $Q_{2}=I_{2}^{2} R_{2} t$, получим: $\quad I_{1}^{2} R_{1} t=I_{2}^{2} R_{2} t \Rightarrow I_{1}^{2} R_{1}=I_{2}^{2} R_{2}$. Применяя, закон Ома для полной цепи, имеем: $E=I_{1}\left(R_{1}+r\right), E=I_{2}\left(R_{2}+r\right)$, тогда $I_{1}\left(R_{1}+r\right)=I_{2}\left(R_{2}+r\right)$, где $I_{1}, I_{2}$ - ток в цепи.

Переходим к системе двух уравнении с тремя неизвестными $I_{1}, I_{2}, r$.

$$
\left\{\begin{array}{l}
I_{1}^{2} R_{1}=I_{2}^{2} R_{2} \\
I_{1}\left(R_{1}+r\right)=I_{2}\left(R_{2}+r\right)
\end{array}\right.
$$

Математическая модель исходной задачи получена - это система двух уравнений с тремя неизвестными.

II этап. Решение задачи в рамках построенной модели. Найдём решение системы.

Для этого запишем уравнения в другом виде:

$$
\left\{\begin{array}{l}
\frac{I_{1}}{I_{2}}=\sqrt{\frac{R_{2}}{R_{1}}} \\
\frac{I_{1}}{I_{2}}=\frac{R_{2}+r}{R_{1}+r}
\end{array}\right.
$$




\begin{tabular}{llllll} 
& ISRA (India) $=\mathbf{3 . 1 1 7}$ & SIS (USA) & $=\mathbf{0 . 9 1 2}$ & ICV (Poland) & $=\mathbf{6 . 6 3 0}$ \\
Impact Factor: & ISI (Dubai, UAE) $=\mathbf{0 . 8 2 9}$ & PUHL (Russia) $=\mathbf{0 . 1 5 6}$ & PIF (India) & $=\mathbf{1 . 9 4 0}$ \\
& GIF (Australia) $=\mathbf{0 . 5 6 4}$ & ESJI (KZ) & $=\mathbf{8 . 7 1 6}$ & IBI (India) & $=\mathbf{4 . 2 6 0}$ \\
& JIF & $\mathbf{1 . 5 0 0}$ & SJIF (Morocco) $=\mathbf{5 . 6 6 7}$ & OAJI (USA) & $\mathbf{0 . 3 5 0}$ \\
\hline
\end{tabular}

Приравнивая $\sqrt{\frac{R_{2}}{R_{1}}}$ и $\frac{R_{2}+r}{R_{1}+r}$, получим: $\sqrt{\frac{R_{2}}{R_{1}}}=$ $\frac{R_{2}+r}{R_{1}+r}$. Возводя в квадрат обе части, имеем: $R_{1}+r$

$\frac{R_{2}}{R_{1}}=\frac{R_{2}^{2}+2 R_{2} r+r^{2}}{R_{1}^{2}+2 R_{1} r+r^{2}} \Rightarrow$

$R_{2} R_{1}^{2}+2 R_{2} R_{1} r+R_{2} r^{2}=R_{1} R_{2}^{2}+2 R_{2} R_{1} r+R_{1} r^{2}$,

$r^{2}\left(R_{2}-R_{1}\right)=R_{2} R_{1}\left(R_{2}-R_{1}\right)$

$\Rightarrow r^{2}=R_{2} R_{1}$, и, наконец, $r=\sqrt{R_{2} R_{1}}$. Решение получено.

III этап - интерпретация. Переведём полученный результат решения математической задачи (системы двух уравнений с тремя неизвестными) на язык исходной задачи. Внутреннее сопротивление $r$ элемента равно $r=\sqrt{R_{2} R_{1}}$. Математическое решение получено, естественно, при условии $R_{2}-R_{1} \neq 0$. Если $R_{2}-R_{1}=0$, т.е. $R_{2}=R_{1}$ из системы получаем $I_{1}=I_{2}$ и $\frac{R_{2}+r}{R_{1}+r}=1$, т.е. $R_{2}+r=R_{1}+r \Rightarrow R_{2}=R_{1}$, т.е. в другой раз спираль включена с тем же сопротивлением $R_{1}$.

Межпредметные связи на уровне знаний в этой задаче раскрыты посредством использования теоретических положений курса физики (закона Ома для полной цепи и т.д.). Эти положения совместно математического аппарата (способа решения системы двух уравнений с тремя неизвестными) приводят к искомому результату.

Ценность такого рода задач в формировании и развитии математического мышления заключается в том, что выявление практической значимости изучаемой математической теории на таких задачах служит мощным стимулом для активизации мыслительных действий студента по совершенствованию математических знаний и самостоятельному, сознательному их приобретению. Мы уверены и наш опыт работы подтверждает это, что решение подобного рода задач является пусть весьма скромным, но творческим актом, поскольку решение задачи предполагает изобретение специально ведущего к поставленной цели рассуждения. Именно этот творческий, исследовательский характер данной задачи более чем что-либо другое привлекает к себе ученика, желающего испытать свой силы и развить укрепляющийся интеллект. В самом деле - как решать систему двух уравнений с тремя неизвестными? Для ответа необходимо проявить нестандартность, не шаблонность мышления, интуицию (конечно, основанную на знании и подкрепленную этим знанием). Догадаться, что можно преобразовать каждое уравнение системы так, чтобы в левых частях получить одно и то же отношение I1 к I2, - это дано не каждому ученику и «озарение» снизу идет не на каждого. Но тот учащийся, который изведал благородную радость творческого достижения, никогда не пожалеет усилий, чтобы вновь ее испытать. Это творческое достижение является для студента стимулом для всё новых и новых напряжений мысли. Именно напряженная, интенсивная мыслительная деятельность учащегося и приводит к положительным результатам в его математическом развитии.

Межпредметные связи могут быть осуществлены не только на уровне знаний, но и на уровне видов деятельности. В математике используются различные виды деятельности в процессе обучения. Например, употребляется вид деятельности составить текстовую задачу по заданному уравнению. Аналогичный вид деятельности может быть применён и в процессе обучения физике.

Межпредметный связь между математикой и физикой на уровне видов деятельности может быть реализована посредством методов научного познания. И в математике, и в физике используются такие методы как наблюдение, сравнение, аналогия, индукция, дедукция, анализ, синтез, обобщение, конкретизация, абстрагирование, специализация.

Как пример, приведём задачу, демонстрирующую реализацию межпредметных связей на уровне деятельности, посредством метода аналогии.

Задача 2: (физическая) Во сколько раз минимально можно уменьшить ёмкость батареи, состоящей из двух параллельно соединённых конденсаторов, при замене параллельного соединения на последовательное соединение?

Решение. Рассмотрим эту задачу как прикладную и найдём её решение по трехэтапной схеме.

I этап. Обозначим через $C_{1}$ и $C_{2}$ - ёмкости отдельных конденсаторов, $C_{n a p}$ - ёмкость батареи при параллельном соединении конденсаторов, $C_{n o c}$ - ёмкость батареи при последовательном соединении. Из курса физики известно, что $C_{n a p}=$ $C_{1+} C_{2} ; \quad C_{n o c}=\frac{C_{1} C_{2}}{C_{1}+C_{2}}$. Составим отношение $\frac{C_{n a p}}{C_{n o c}}=\frac{\left(C_{1}+C_{2}\right)^{2}}{C_{1} C_{2}}$. Требуется узнать, при каких значениях $C_{1}$ и $C_{2}$ значение отношения $\frac{C_{n a p}}{C_{n o c}}$ будет наименьшим. Математическая модель построена. 


\begin{tabular}{llllll} 
& ISRA (India) $=\mathbf{3 . 1 1 7}$ & SIS (USA) & $=\mathbf{0 . 9 1 2}$ & ICV (Poland) & $=\mathbf{6 . 6 3 0}$ \\
Impact Factor: & ISI (Dubai, UAE) $=\mathbf{0 . 8 2 9}$ & PUHL (Russia) $=\mathbf{0 . 1 5 6}$ & PIF (India) & $=\mathbf{1 . 9 4 0}$ \\
& GIF (Australia) $=\mathbf{0 . 5 6 4}$ & ESJI (KZ) & $=\mathbf{8 . 7 1 6}$ & IBI (India) & $=\mathbf{4 . 2 6 0}$ \\
& JIF & $\mathbf{1 . 5 0 0}$ & SJIF (Morocco) $=\mathbf{5 . 6 6 7}$ & OAJI (USA) & $\mathbf{0 . 3 5 0}$ \\
\hline
\end{tabular}

II этап. Решим задачу в рамках построенной модели. В курсе математики используется неравенство $a+\frac{1}{a} \geq 2$, где $a>0$. Минимальное значение равно 2. Воспользуемся методом аналогии и преобразуем отношение $\frac{C_{n a p}}{C_{n o c}}$ к виду: $\frac{C_{n a p}}{C_{n o c}}=\frac{\left(C_{1}+C_{2}\right)^{2}}{C_{1} C_{2}}=\frac{C_{1}^{2}+2 C_{1} C_{2}+C_{2}^{2}}{C_{1} C_{2}}=\frac{C_{1}}{C_{2}}+\frac{C_{2}}{C_{1}}+2 \geq 4$.

Наименьшее значение $\frac{C_{n a p}}{C_{n o c}}$ равно 4. Видим, что оно достигается при $C_{1}=C_{2}$.

III этап (интерпретация). Переведём полученный результат на язык исходной задачи. Поскольку наименьшее значение $\frac{C_{n a p}}{C_{n o c}}=4$, достигается при $C_{1}=C_{2}$ (ёмкости конденсаторов одинаковы), то при замене параллельного соединения на последовательное соединение можно минимально уменьшить ёмкость батареи в 4 раза.

В этой задаче использование аналогии между математическими величинами и величинами физическими существенно помогло получить требуемый результат. Обнаружение сходства разных явлений, пусть даже и совсем неглубокого, способствует активизации мышления, ибо прежние знания (о математических величинах: ( $a$ $+1 / a)>2$, где $a>0$ ) выступают в новом свете применительно к физическим величинам $\left(C_{1} / C_{2}+\right.$ $C_{2} / C_{1}>2$ ).

Умение находить аналогии между утверждениями - это доказательство аналогии. Если замечается аналогии теорий - это высшая инстанция научного познания. Так что действительно, среди ценностей интеллекта одно из важнейших мест занимает умение находить и применять аналогии. В рассмотренной задаче, проводя умозаключение по аналогии, учащийся совершает сложный мыслительный процесс, в котором в единстве и взаимопроникновении применяются приёмы анализа и синтеза. Здесь математические объекты и объекты физические запечатлеваются в сознании ученика не изолированно друг от друга, а в тесной связи друг с другом, в единстве. Таким образом прикладные задачи могут быть с успехом использованы в качестве средства формирования и развития математического мышления учащихся.

\section{Conclusion}

Подводя итог исследованию развития математического мышления при реализации межпредметных связей в процессе решения прикладных задач, приходим к выводам:

1. Прикладные задачи, решаемые на основе использования межпредметных связей, способствуют более глубокому пониманию учащимися идеи материальности мира;

2. В процессе нахождения решения прикладной задачи с использованием межпредметных связей формируется и активно развиваются такие качества, как математическое мышление, которое способствует к обобщению, абстрагированию, умение анализировать, синтезировать, строить суждения и умозаключения, схематизировать, что приводит к развитию гибкости и глубины мышления, его оригинальности и рациональности.

3. Использование в учебном процессе прикладных задач с установлением при их решении межпредметных связей способствует более глубокому усвоению учащимися, как математики, так и смежных дисциплин (физики и др.).

4. Прикладные задачи приучают школьников видеть в задачах пространство их учебных действий, источник информации об их способностях, ситуацию, в которой можно ощутить приятное и весьма вдохновляющее чувство собственной компетенции, что вполне согласуется с требованиями гуманизации математического образования.

\section{References:}

1. Babaev, D., Haitov, S. K. (2017). Idei problemno-razvivajushhego obuchenija na lekcijah po fizike $\mathrm{v}$ tehnicheskih universitetah [Tekst]. INTERNATIONAL ACADEMY JOURNAL Web of Scholar. RS Global Sp. z O.O. Warsaw, Poland. - November 2017. - № 8(17), pp. 24-29.
2. Isakov, A.I. (2017). Formirovanie kvantovyh predstavlenij $v$ obshheobrazovatel'noj shkole na osnove principa preemstvennosti [Tekst]. Nauchno-metodicheskie innovacionnogo problemy obrazovanija: Sbornik nauchnyh trudov. V $2 \mathrm{ch}$. Ch. 1. Saratov, pp.168-171. 


\begin{tabular}{llllll} 
& ISRA (India) $=\mathbf{3 . 1 1 7}$ & SIS (USA) $=\mathbf{0 . 9 1 2}$ & ICV (Poland) & $\mathbf{= 6 . 6 3 0}$ \\
Impact Factor: & ISI (Dubai, UAE) $=\mathbf{0 . 8 2 9}$ & PUHЦ (Russia) $=\mathbf{0 . 1 5 6}$ & PIF (India) & $=\mathbf{1 . 9 4 0}$ \\
& GIF (Australia) $=\mathbf{0 . 5 6 4}$ & ESJI (KZ) & $\mathbf{8 . 7 1 6}$ & IBI (India) & $=\mathbf{4 . 2 6 0}$ \\
& JIF & $\mathbf{1 . 5 0 0}$ & SJIF (Morocco) $=\mathbf{5 . 6 6 7}$ & OAJI (USA) & $\mathbf{0 . 3 5 0}$ \\
\hline
\end{tabular}

3. Kashlev, S. S. (2011). Interaktivnye metody obuchenija. (p.224). Minsk: Tetra Sistems.

4. (2017). Nacional'naja programma «40 shagov na puti k procvetaniju» 2018-204 gg. [Tekst]: Postanovlenie ZhK Kyrgyzskoj Respubliki №1836-VI ot 25.08.2017.
5. Smolkin, A. M. (2003). Metody aktivnogo obuchenija [Tekst]. (p.150). Moscow: Vysshaja shkola.

6. (2012). Strategija razvitija obrazovanija v Kyrgyzskoj Respublike na 2012-2020 gody [Tekst]: utv. postanovlenie Pravitel'stva Kyrg. Resp. ot 23 marta 2012 g., № 201 // Normativ. akty Kyrg. Resp. № 29, pp.2-54. 